\title{
THE TPC TRIGGER FOR THE DELPHI EXPERIMENT
}

G. Darbo and B.W. Heck

CERN, Geneva, Switzerland

\section{Abstract}

A hardware trigger has been designed for the Time Projection Chamber of the DELPHI Experiment. It will have two different levels, a fast and simple one, and a second one which is more precise. On the first level, a system of shift registers is used to search for track pieces in less than 1 us. The second level uses a specialized track finding device (the "Contiguity Processor") with a highly parallel structure. It follows tracks through the TPC in about $8 \mu$ s after the drift time. The use of specially designed gate arrays permits to build a system with high granularity at reasonable complexity and cost, which also will provide a good backgroun rejection. In this report, we describe briefly the general trigger situation in the experiment and then the principle ideas of the two TPC trigger systems. In addition, we describe the technical implementation of both systems.

\section{Introduction}

DELPHI is one of the experiments at the Large Electron Positron (I.EP) collider, currently being constructed at CERN. The detector for the experiment has been described in a technical proposal [1]. The main tracking device for high energy charged particles in this detector is a Time Projection Chamber (TPC) which measures accurately the position of the particles' flight path in three dimensions. The chamber has the form of a cylindrical tube of more than $2 \mathrm{~m}$ in length and diameter (fig. l) and is filled with a drift gas. Charged particles produce in this volume ionization along their trajectories. The electrons are drifted parallel to the beam direction under the influence of an electric field away from the central high-voltage plane towards either of the two end-plates. There the sensitive elements (about 2300 sense wires and 20000 cathode pads) are installed to detect the electrons. The pads measure the position information in the plane perpendicular to the drift direction, while the axial position is determined by the drift time.

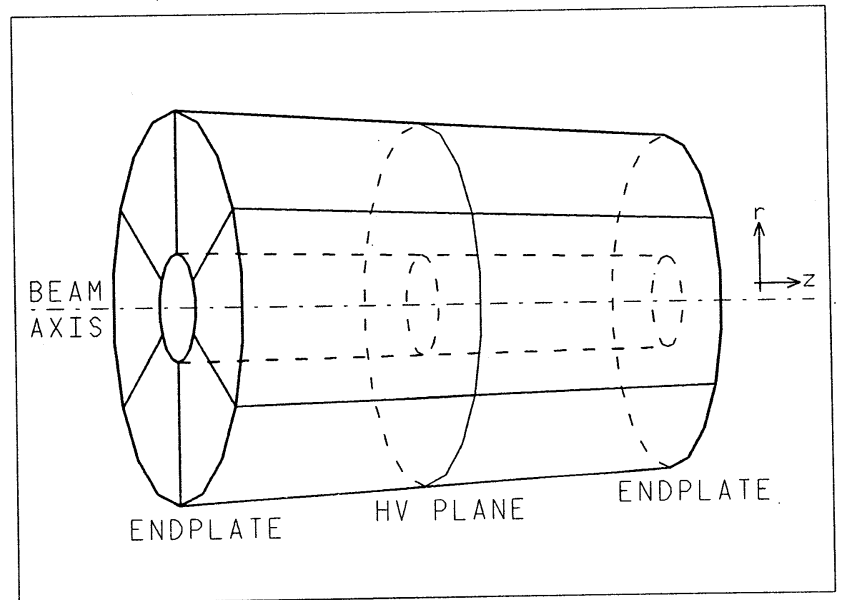

Fig. 1 The Time Projection Chamber (TPC).

The online event selection procedure for the experiment can be described as a sequence of four different processes, which take more and more time while also becoming more and more selective and precise. The first two levels are foreseen for hardware triggers and the last two for software processes. Only the first two will be treated in this report. The original idea of the "Contiguity Processor", whose principle will be used on the second trigger level, is described in refs [2,3]. Some more recent details are given in [4,5]. The current status will be described in this report.

The I-EP collider will produce one beam cross-over in the centre of the detector roughly every $22 \mu \mathrm{s}$. The trigger system has to examine each of the events and to decide, whether it fulfils the general trigger requirements of the experiment: the events must either contain at least one track of a charged particle coming from the vertex and having a transverse momentum of more than $1.5 \mathrm{GeV} / \mathrm{c}$, or have deposited sufficient energy in one of the calorimeters. However good events of this type are expected only at a rate of 1 event per second or less. On the other hand, background events are expected at a rate around $1 \mathrm{kHz}$, which makes a powerful rejection scheme necessary. Even then, a certain fraction of these background events will not be rejected by the trigger. In this case it will have to accept the event provisionally and leave the final decision to the off-line analysis.

A time of $3 \mu$ s is foreseen for taking a decision on the first trigger level. This level is supposed to reduce the rate to about $1 \mathrm{kHz}$. If the decision is negative, the event will be aborted and the gate for drift devices like the TPC will be closed until the next beam cross-over. This is foreseen in order to reduce the amount of space charge due to positive ions in the drift volume.

In the case of a positive decision, the following beam cross-over will be ignored and the second trigger level will have about $40 \mu \mathrm{s}$ time for a more refined decision. Only in case of a positive decision on this level, the information in the front-end buffers will be read out. Otherwise the event will be overwritten. We expect a rate of about $20 \mathrm{~Hz}$ at the output of this level.

In this trigger scheme, the TPC plays an important part. because it can cover the region $15^{\circ} \leqq \theta \leqq 165^{\circ}$. However, due to time limitations the first level TPC trigger covers only the forward region, $15^{\circ} \leq \theta \leq 40^{\circ}$ and $140^{\circ} \leq \theta \leq 165^{\circ}$, which will even be complemented further by the information from drift chambers. The other part $\left(35^{\circ} \leqq \theta \leqq 145^{\circ}\right)$ will be covered by the TPC on the second trigger level only, after the inner and outer detectors have prepared this decision already on the first level.

\section{The First Level Trigger}

The region of the first level track trigger is shown in an $r z$ projection of the TPC in fig. 2. The system searches for track pieces in a trigger region close to the plane of the 192 sense wires which are perpendicular to the drawing. In total $1.6 \mu$ s of drift time will be used, which corresponds to about $10 \mathrm{~cm}$ in drift direction. The angular acceptance is surrounded by the wire plane and the dashed lines.

As will be described below, the trigger is neither sensitive to individual hits nor random tracks but only to tracks which come approximately from the interaction point and are more or less straight in this $\mathrm{rz}$ projection. In addition it will not be sensitive to inefficiencies of individual wires in the TPC, since it uses always groups of wires. Finally, the trigger will be very fast in order to allow to combine its results with the trigger results from other drift chambers within the time limit of $3 \mu s$. 


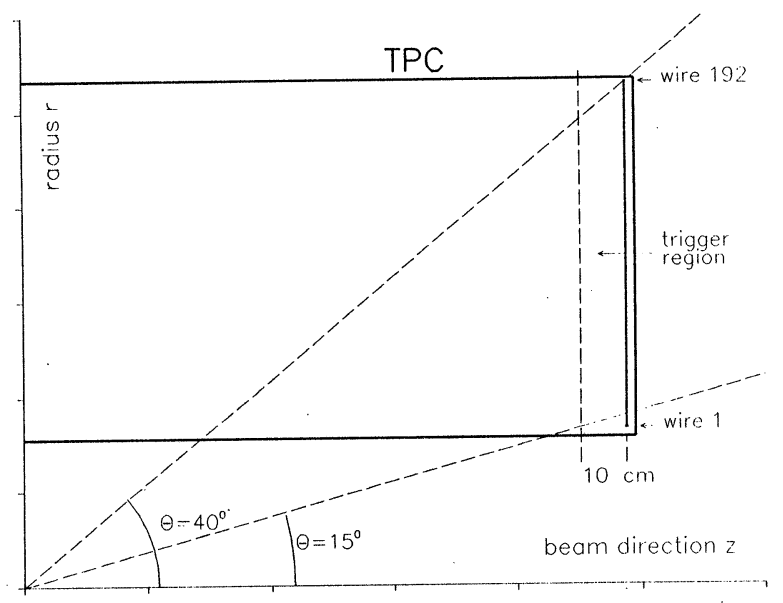

Fig. 2 The trigger region of the first level trigger, between the wire plane and the dashed lines.

\section{The Implementation}

The trigger uses TPC wire signals, which are converted into digital information by means of discriminators with adjustable threshold. Out of the 192 wires in each TPC sector 64 groups of signals will be formed. The number of signals per group however will not be constant but will be two wires per group at small radii and then rise up to five wires per group at the outermost wires. The reason for this choice will be given below. The 64 signals will be fed into 16-bit serial-input shift registers with a clock of $10 \mathrm{MHz}$. The parallel outputs of these shift registers now represent a two-dimensional picture of the tracks in $r-z$ projection in the TPC directly after the beam-beam interaction: the 16 bits in each register correspond to the $z$ coordinate, and the 64 registers to the different radial positions $r$.

Fig. 3 shows how the outputs of the shift registers are connected to the inputs of the 4-fold AND circuits. Only 4 out of the 64 registers are shown. A track piece in the TPC will only be accepted, if it fulfils one of the AND conditions.

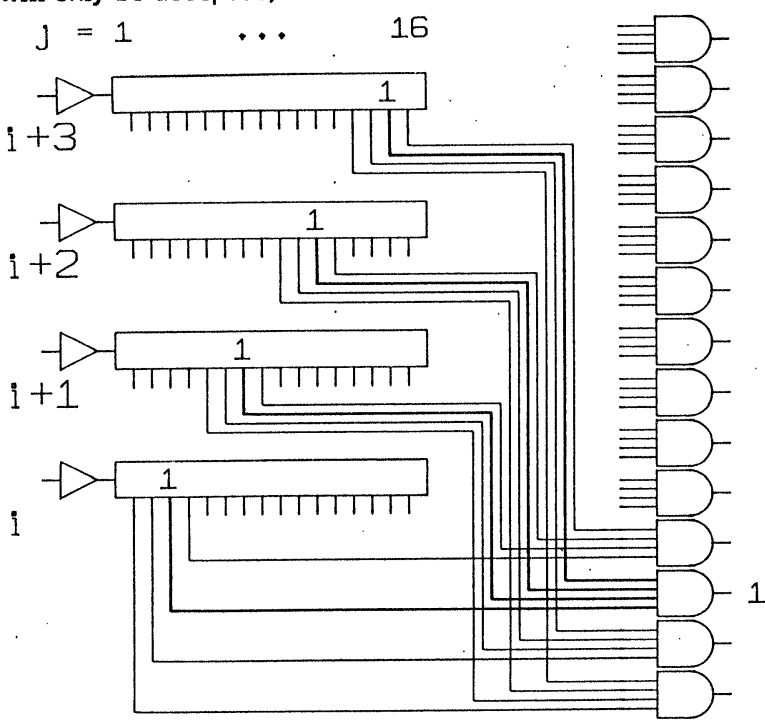

Fig. 3 Shift registers and ANDs for the first level trigger.

Let $\mathrm{i}, 1 \leqq \mathrm{i} \leqq 64$, be the number of the shift register, and $j, 1 \leqq j \leqq 16$, be the index of a bit in a shift register, then the bits themselves are described by $b_{i j}$. Using this terminology the tracks which will be accepted by the trigger must have the following bits set to one:

$$
b_{i j}, b_{i+1, j+4}, b_{i+2, j+8} \text { and } b_{i+3, j+12} \text {. }
$$

The example in fig. 3 is given for

$$
b_{i, 3}, b_{i+1,7}, b_{i+2,11} \text { and } b_{i+3,15} \text {. }
$$

As was mentioned before, the contents of the shift registers is a mapping of the track in the TPC into the plane of the shift registers. The bits, which are set to 1 , correspond to samples of the tracks. A hypothetical line in fig. 3 which connects these bits in the registers, represents therefore a track in the TPC. There this track has a certain inclination angle $\theta$, which is a function of the radial position of the track on the sense wire plane. As an example, a track near wire 1 l has $\theta=15^{\circ}$, while it has $\theta \simeq 40^{\circ}$ close to wire \# 192. If one would group the wire signals, in a radius independent fashion, then the cabling between shift registers and ANDs would become dependent on $\theta$. However, by grouping them into small groups at small radii and large ones at large radii, this dependence is eliminated. The circuit will be then uniform and much simpler.

Up to now we have assumed that a track produces only one hit in a shift register. This is however an approximation. In reality the analog signals at the input of the discriminators have a minimum pulse width of approximately $100 \mathrm{~ns}$. Especially if the tracks are inclined, these pulses will become longer. Therefore it will happen often that two or more hits per track will be generated in the shift registers. This effect will reduce the influence of inefficient wires in the TPC even more.

\section{The Second Level Trigger}

The second level track trigger searches again for tracks of charged particles, but this time in the region $35^{\circ} \leqq \theta \leqq 145^{\circ}$, which overlaps slightly with the region of the first level trigger. The two other dimensions of the trigger region are shown in fig. 4: the $r \Phi$ projection of a TPC end-plate. Each of these end-plates is made of six identical sectors subtending $60^{\circ}$ in azimuthal angle. The cathode pads, which were already mentioned, are located on these sectors. They form 16 concentrical circles around the beam axis. The size of a pad is about $0.5 \mathrm{~cm}^{2}$. The number of pads per row increases with the radius of the circle: there are 64 pads on the innermost and 144 pads on the outermost pad row of each sector. The pulses induced on the pads will be used to measure accurately the position of the tracks in the drift volume. They are also used in the second level track trigger.

Fig. 4 shows also a computer simulated event with roughly 25 tracks, which are indicated by the dashes at those places, where they have generated a signal on the pads.

In the following we describe the trigger processor, which will search for this kind of tracks, but during the presence of a yet unknown amount of background in form of tracks not coming from the vertex and of uncorrelated noise in the detector. Even though the processor could in principle deal with the entire azimuthal range $0^{\circ} \leqq \phi \leqq 360^{\circ}$, it is now designed in a form which will process data sector by sector. The reason for this is the so-called intersector gap, a narrow dead zone between two sectors due to the mechanical structure, which is not equipped with pads. It will be very difficult to follow tracks crossing this gap in a hardwired processor, since they loose $50 \%$ or more of its digitizations there, if they fulfil the trigger requirement with respect to the transverse momentum condition mentioned above.

\section{The Principle of the Contiquity Processor}

Consider a rectangular mesh as shown in fig. 5 . The small dots represent latches, which are reset to zero. The large dots are latches set to one. These latches are connected to the sensitive cells of a 2-d detector, and the pulses from this detector will control the state of the 
latches. In the example of fig. 5 there were two tracks going through the detector from the bottom to the top, leaving behind some ionization, which finally set the corresponding latches. The task of the trigger processor might now be to find out whether there were tracks in the detector or not. This is achieved with the help of the vertical and horizontal switches between neighbouring cells.

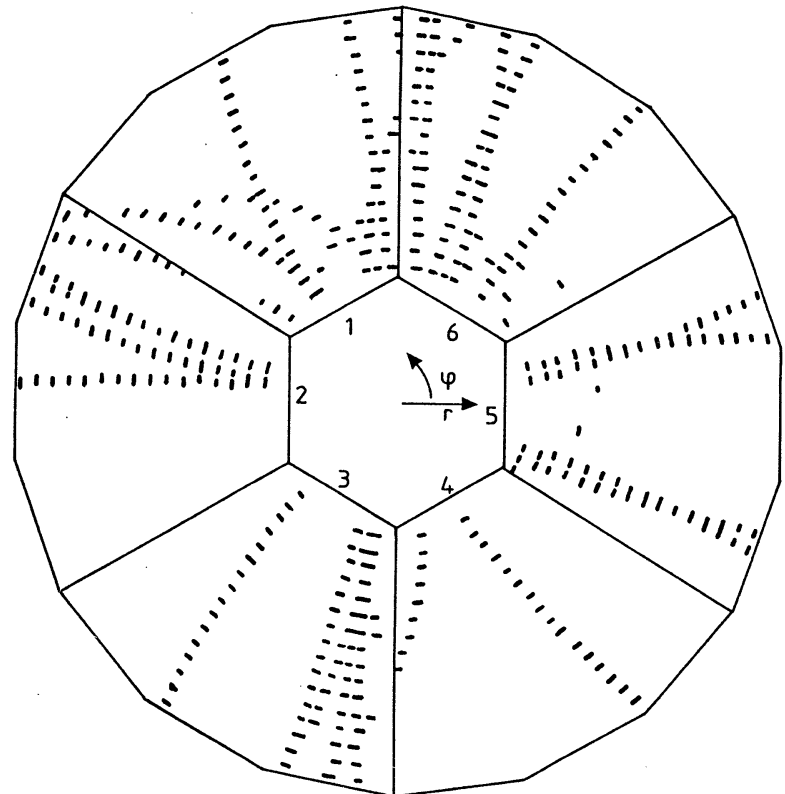

Fiq. $4 \mathrm{~A}$ simulated event in the $\mathrm{r} \phi$ projection.

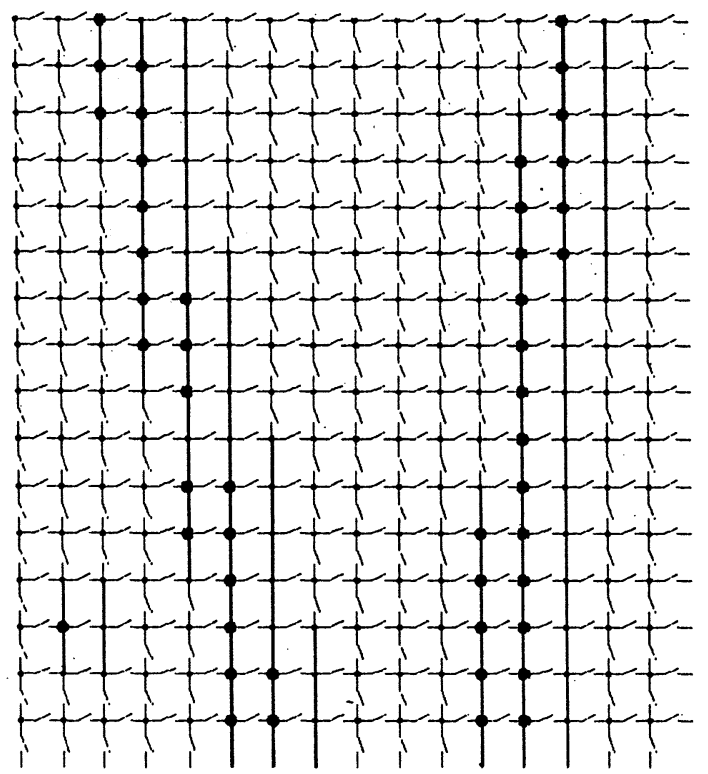

Fig. 5 The Contiguity Processor Array.

As can be seen, some of the switches around the tracks are closed. There is even one vertical row of switches (the 3rd row from the right), where all switches from top to bottom are closed. If one applies now a certain voltage $v$ at the bottom of all rows, then it is transmitted through the matrix up to the top. When counting the number of times this voltage $v$ is present at the top, one finds in this example exactly one, which means there was a track passing through the detector which was sufficiently stiff to be accepted by the trigger. The track on the left hand side however did not produce such a contiguous path through the matrix, and is therefore not detected. It is because of this principle that the trigger system has the name Contiguity Processor.

\section{The Connection Memories}

The latches, which have been described in the previous paragraph, form the "Image Memory" (IM). There are two more memories, the "Vertical" (VCM) and the "Horizontal Connection Memory" (HCM). All memories have the same size. The set of three memory cells, one from each type (IM, VCM and HCM) form together with the interconnecting logic a "node" of the network. The VCM controls the state of the vertical switch between this node and its neighbour below and the HCM the state of the horizontal one leading to the right. If a bit in the connection memory is set, the corresponding switch is closed and vice versa. The connection memories are loaded from the contents of the IM under control of the processor:

The two copy instructions "Copy to HCM" (COH) and "Copy to VCM" (COV) copy the contents of the IM into the connection memories HCM or VCM respectively. For example, the instruction COV copies the state of the IM into the VCM, node by node.

- The shift instructions will shift the information in a connection memory in one of the four directions (up, down, left or right) by one position. This is applicable for either memory (HCM and VCM). There are even two modes: in the first one ("shift only") the operation is completely like in a normal shift register. In the second one ("shift with feedback") a " 1 " bit which is shifted out of one node will be fed back into the same node, again during the same cycle.

All these instructions do operate on all nodes of the bidimensional array at the same time. By using several instructions one after the other one can build up some special patterns, which have different features. One simple case would be to use only Copy Vertical, which would close only the switch below the node. This pattern would be useful in cases where the tracks are all vertical.

The programmability of these patterns is one of the features of the Contiguity Processor. We want to mention only three examples:

- Some patterns will select only tracks with a certain curvature, say left or right. Fig. 6(a) gives an example, which could be used to select particles with a certain charge. It may be useful to apply this left handed pattern first and in a second step the symmetrical right handed one, and achieve a different result than when using a "T"-shaped pattern immediately.

- Some other patterns make the processor less sensitive with respect to random inefficiencies of the detector. Assume one individual node is often not giving a signal. If the track passes through this node, then a pattern with two vertical switches, which are vertically neighbours will still allow to detect the track correctly (fig. 6(b)).

- The pattern in fig. 6(c) has the feature of limiting the amount of curvature for the accepted tracks. This corresponds to a limit on the transverse momentum of the particles.

The final patterns to be used in the experiment will depend on the background conditions and on physics requirements. But it will most likely be a combination of some of the simple patterns shown. 


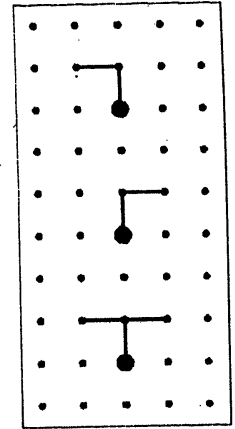

a) left handed
right handed

$T$ shoped

Fig. 6 Different kinds of connection patterns

\section{The Mapping}

To relate the actual TPC and the Contiguity Processor, the signals from the cathode pads will be used. Since we need much less signals than pads, we can group the pad signals from different neighbours together. Fig. 7 shows schematically how the pad groups will look like in a certain region of one sector. The grouping is done such, that there are always 24 groups in one row and sector thus covering an angular range of $\Delta \phi=2.5^{\circ}$. This means that the groups become wider with increasing radius. The 24 groups in the 16 pad rows can be seen as a matrix, which maps directly onto the rectangular matrix of the mesh with the nodes described in the previous paragraph.

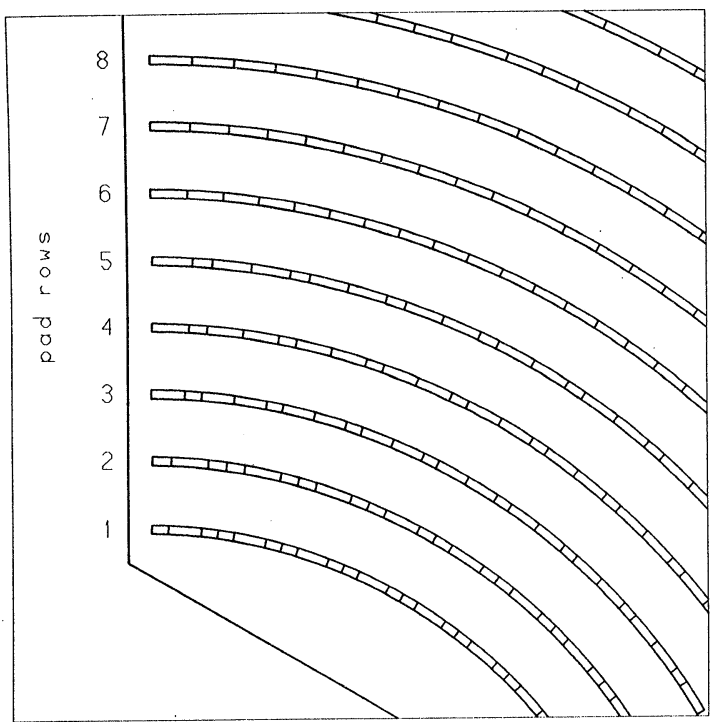

Fig. 7 Part of a sector plate with the pad groups.

Electrically, the connection between pads and nodes in the contiguity processor is done in the following way: the output signals from the pads are digitized. The grouping is then performed in a similar way as in the first trigger level by ORing open collector discriminator outputs on the backplane of the crates. These signals are then connected to the inputs of the IM of the processor.

\section{The 3-d Processor}

The processor as described up to now has one memory location in its IM for each node. There is no recording of the drift time foreseen. Therefore it works only in the 2 dimensions $\mathrm{r}$ and $\phi$. In the 3-d processor, which will be implemented in the TPC trigger, the memory has 5 locations: $I_{\mathrm{i}}$ with $\mathrm{i}=1, \ldots, 5$. Each memory is foreseen for a different drift time range. Fig. 8 , which represents again a projection of the TPC in $r z$, shows 5 different slices $C_{i}$. Each of them is $\Delta \theta=11^{\circ}$ wide, and covers all the pad rows, except $C_{1}$, which cannot cover row 16 completely. The signals from the pad groups can now be distributed to the different $I M_{i}$ according to their drift time. Whatever falls into a time window corresponding to $C_{i}$, will be loaded into $I M_{i}$.

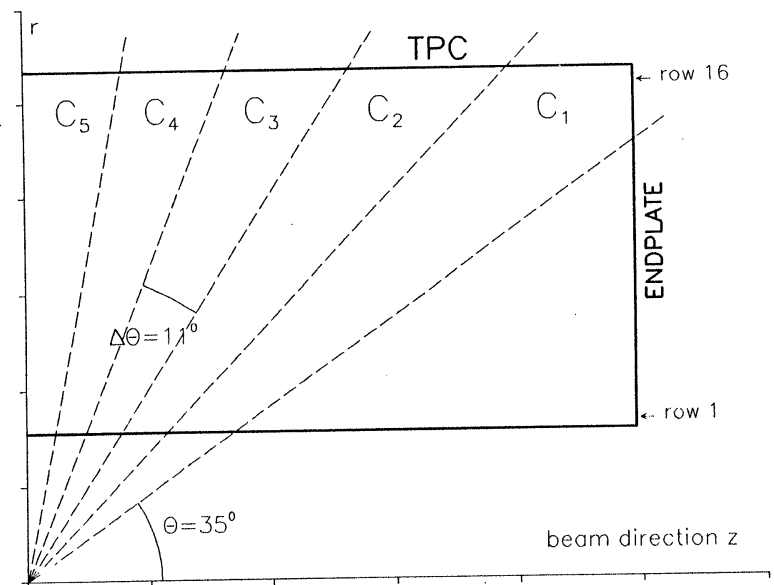

Fig. 8 The five slices $C_{i}$ for the 3-d processor.

This principle relies on the fact, that trajectories of particles in the $\mathrm{rz}$ projection are not strongly influenced by the axial magnetic field. They are in first approximation straight and will mostly fall into only one slice. If however the slices are constructed with a narrow overlap in $\theta$ between neighbours, then even trajectories on border lines will fall completely into one of the two slices, where they can be detected. On the other hand, the overlap must not be too big, because these tracks would then sometimes be detected and counted twice.

This 3-d processor with 5 image memories has however only one vertical and one horizontal connection memory. After all IMs have been loaded during the drift time, the processor starts working on the data in $\mathrm{IM}_{1}$, preparing VCM and HCM and searching for a track. After having stored the result, it will clear VCM and HCM again and repeat the procedure for the next IM. In this way it will step through all 5 memories. Since the processing for one slice takes only about $1.5 \mu \mathrm{s}$, the total processing time is less than $8 \mu \mathrm{s}$ for all 5 slices.

The 3-d processor offers mainly two advantages with respect to the simpler $2-d$ version:

- Since it covers only $11^{\circ}$ in the $\theta$ direction during one processing cycle, the image memory will contain less data. Therefore the chance of combining random background hits and accept them as a track is very much reduced.

- Good tracks will come from the vertex at $z=0$ and an uncertainty of $\pm 5 \mathrm{~cm}$. Unwanted tracks however will come from any direction and also from the beam pipe at large values of $z$. They should be rejected. This is easier when having 5 narrow slices than only one broad one. 
Fig. 9 shows schematically the timing conditions for loading the $5 \mathrm{IMs}$ as a function of time and for 3 different pad rows. At the beginning of the drift time $t=t_{0}, I M_{1}$ of row 16 will be enabled as shown at the top left $(A)$. After some time, $\mathrm{IM}_{2}$ of the same row will be enabled and $\mathrm{IM}_{2}$ disabled (B). While $\mathrm{IM}_{2}$ is still enabled, $\mathrm{IM}_{2}$ of row 8 will be enabled (C) too. In the example of fig. $9 \mathrm{IM}_{2}$ of row 16 and $\mathrm{IM}_{2}$ of row 8 will be disabled at the same time (D) and so on. Just before reaching the maximum drift time, $\mathrm{IM}_{5}$ of all rows is enabled.

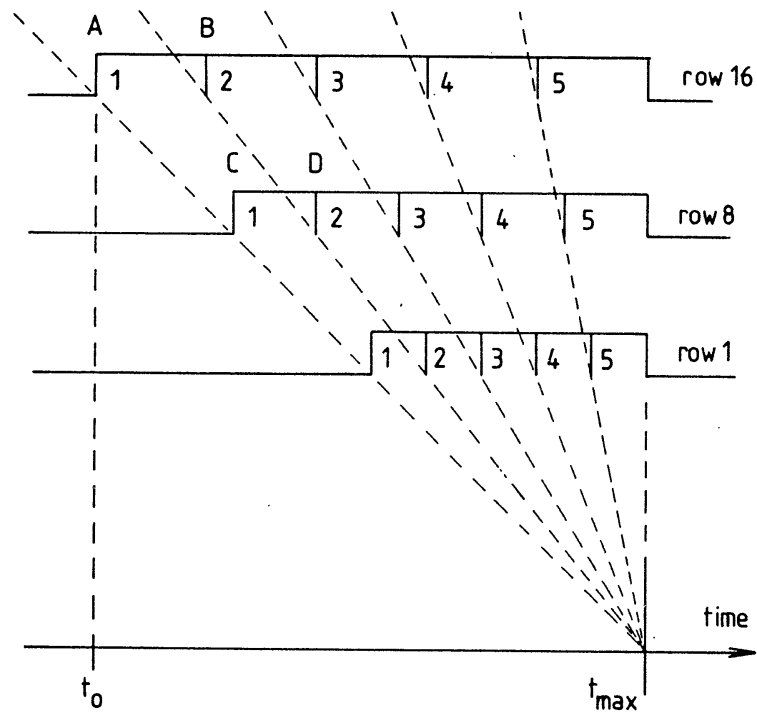

Fig. 9 The scheme for distributing the detector signals to the individual memories.

The generation of these gating signals will be done with a minimum step of $100 \mathrm{~ns}$, which corresponds to $6 \mathrm{~mm}$ in the TPC. The overlap between consecutive slices is programmable in the same way, even if this is not shown in fig. 9 for reasons of clarity.

\section{Post processing and implementation}

As described earlier, the data from the IM are used to set up the switch pattern in VCM and HCM. Afterwards it will be tested, whether or not there is a path along the switches from the bottom to the top through the contiguity array (fig. 5). The result is a pattern of as many bits as the number of columns in the array. It will be stored as an intermediate result and will be used on the third trigger level, which is foreseen as a programmable micro-processor. However, the detailed information in these patterns is not needed for the decision on the second trigger level. Therefore an ORing of 6 signals into 1 will generate the pattern to be given to the central decision logic, which combines results from the TPC with results from other detectors. This pattern contains 24 bits for the $\phi$ coordinate times 10 bits for the $\theta$ slices (the two halves of the TPC have each 5 bits, as described above). These numbers have been chosen in accordance with the general DELPHI trigger requirements.

The regular structure of the contiguity array is ideally implemented in semi-custom integrated circuits because of the large number of identical nodes. A Gate Array chip is being designed for a matrix of 4 times 4 nodes, where each node contains 5 image memories, both connection memories, the contiguity network, the data distribution part and the instruction decoder. 24 of these chips will be needed for one Fastbus card, which will be equipped in addition with a memory for data and instructions. The entire system for the
TPC will need 2 crates, each of them with a control module and a readout module. The control module is connected with the six processors in the crate via a special bus.

\section{The rz Trigger}

For redundancy, the TPC group will implement an additional trigger on the second level, which has been proposed by our colleagues. It provides a somewhat larger granularity in $\theta$ but substantially lower granularity in $\phi$. This $\mathrm{rz}$ trigger is described elsewhere [6]

\section{Conclusion}

The track trigger for the TPC as described is composed of one forward part, based on signals from wires and one central part, which uses pad signals. These parts complement each other in order to cover finally the full acceptance of the $\operatorname{TPC} 15^{\circ} \leqq \theta \leqq 165^{\circ}$.

The first level part is looking for track pieces of about $10 \mathrm{~cm}$ length. It makes a 4-fold coincidence between hits of a track. Hence it is insensitive to individual spurious hits or tracks from far away of the interaction point. It is also not sensitive to inefficiencies of individual wires, because it uses always more than 1 wire in a group. The logic is very uniform and fast. It therefore allows to combine the TPC decision with the results from other drift chambers within the $3 \mu$ s decision time.

The Contiguity Processor searches in the TPC for tracks in 3 dimensions on the second trigger level. The fine granularity in $\Phi$ and the 10 individual slices in $\theta$ will make the processor rather insensitive with respect to noise and background from the LEP machine. It will be able to reject tracks from beam-wall interactions, which miss the beam-beam interaction point by far. Even though it processes data from 4600 nodes, it is very fast due to its parallel structure.

\section{Acknowledgements}

We are indebted to Professor S. Vitale and Dr. L. Rossi for the help they have given us during the early phase of this project. We are grateful for the continuous support of Drs H. Wenninger and H.J. Hilke. It is also a pleasure to acknowledge stimulating discussions on this subject with our friends from the TPC group, and especially Drs. J.V. Allaby and J.E. Augustin.

\section{References}

[1] DELPHI Technical Proposal, CERN/LEPC 83-3, LEPC/P 2, 17 May 1983.

[2] G. Darbo, maschere di contiguità e loro applicazione nella ricostruzione di tracce di particelle in tempo reale, thesis Università di Genova (1980).

[3] G. Darbo and S. Vitale, A second stage trigger for track reconstruction based on contiguity ideas, Nucl. Instr. and Methods 190 (1981) 81-88.

[4] G. Darbo, B. Heck and L. Rossi, A three-dimensional ripple trigger for the DELPHI Time Projection Chamber Paper presented at the Time Projection Chamber Workshop, Vancouver, B.C., Canada, June 1983 and AIP Conference Proceedings No. 108.

[5] G. Darbo and B.W. Heck, The Contiguity Trigger for the DELPHI TPC, presented at the 2nd Topical Seminar on Perspectives for Experimental Apparatus at Future High-Energy Machines, 5-9 Ma 1986, San Miniato, Italy.

[6] D. Delikaris, Simulation d'un trigger rapide ..., thesis, Université de Paris-Sud, 1986. 
